\title{
CHAPTER 3: EQUILIBRIUM FRACTIONATION OF NON-TRADITIONAL STABLE ISOTOPES: AN EXPERIMENTAL PERSPECTIVE
}

\author{
Anat Shahar ${ }^{1}$, Stephen M. Elardo ${ }^{1}$ and Catherine A. Macris ${ }^{2}$ \\ ${ }^{1}$ Geophysical Laboratory, Carnegie Institution for Science, Washington DC 20015 \\ ${ }^{2}$ Indiana University - Purdue University Indianapolis, IN 46202
}

\section{INTRODUCTION}

In 1986, O'Neil wrote a Reviews in Mineralogy chapter on experimental aspects of isotopic fractionation. He noted that in order to fully understand and interpret the natural variations of light stable isotope ratios in nature, it was essential to know the magnitude and temperature dependence of the isotopic fractionation factor amongst minerals and fluids. At that time it was difficult to imagine that this would become true for the heavier, so called non-traditional stable isotopes, as well. Since the advent of the multiple collector inductively coupled plasma-source mass spectrometer (MC-ICP-MS), natural variations of stable isotope ratios have been found for almost any polyisotopic element measured. Although it has been known that as temperature and mass increase, isotope fractionation decreases very quickly, the MC-ICP-MS has revolutionized the ability of a geochemist to measure very small differences in isotope ratios. It was then that the field of experimental non-traditional stable isotope geochemistry was born. As O'Neil (1986) pointed out there are three ways to obtain isotopic fractionation factors: theoretical calculations, measurements of natural samples with well-known formation conditions, and laboratory calibration studies. This chapter is devoted to explaining the techniques involved with laboratory experiments designed to measure equilibrium isotope fractionation factors as well as the best practices that have been learned. Although experimental petrology has been around for a long time and basic experimental methods have been well-refined, there are additional considerations that must be taken into account when the goal is to measure isotopic compositions at the end of the experiment. It has been only about ten years since these initial studies were published, but much has been learned in that time about how best to conduct experiments aimed at determining equilibrium fractionation factors. We will not focus on the scientific results that have been determined by such experiments, as each chapter in this book will focus on a different element of interest. Instead we will provide a how-to for those interested in conducting these experiments in the future.

\section{FACTORS INFLUENCING EQUILIBRIUM FRACTIONATION FACTORS}

Equilibrium isotope fractionation is driven by the effects of atomic mass on bond vibrational energy. The relationships are easily understood using a simple molecule as an example. All molecules have a zero-point vibrational energy (ZPE) $=1 / 2 h v$, where $v$ is the vibrational frequency and $h$ is Planck's constant. The vibrational frequency of a particular mode for a molecule can be approximated using Hooke's Law, $\nu=1 / 2 \pi \sqrt{ }(\mathrm{k} / \mu)$, where $\mathrm{k}$ is the force constant and $\mu$ is the reduced mass of the molecule (e.g. $\mu=m_{a} m_{b} /\left[m_{a}+m_{b}\right]$, where $m_{a}$ and $m_{b}$ are the atomic weights of two atoms in a diatomic molecule). When a light isotope in a molecule is substituted by a heavy isotope the potential energy curve does not (to a first approximation) change shape, hence the force constant does not change, but the vibrational frequency does change. When a more massive isotope is substituted, the reduced mass of the molecule increases,

This is the author's manuscript of the article published in final edited form as: 
which decreases the vibrational frequency and the energy. Therefore, equilibrium stable isotope fractionations are quantum-mechanical effects that depend on the ZPE of the molecule being investigated.

The same principle applies to crystalline materials. To a first approximation, the most important factor that determines the magnitude of isotopic fractionation is differences in bond strength; stiffer bonds concentrate the heavy isotope. Bond strength (stiffness) determines vibrational frequency and vibrational frequency determines internal energy. Stiffer bonds tend to correlate with high oxidation state, covalent bonding, and low coordination number. Therefore, all else being equal, the heavy isotopes of an element will partition preferentially into phases with these characteristics. Furthermore, increasing the pressure experienced by a given phase stiffens the bonds while increasing the temperature weakens the bonds. Therefore, the main variables that influence the equilibrium fractionation factor between two phases are temperature, pressure, oxygen fugacity and composition, as these are the variables that will most notably change the bond strength of the isotope of interest. For a thorough explanation of how temperature, pressure, composition and oxygen fugacity affect isotope fractionation the interested reader can reference Young et al. (2002; 2015) and Schauble (2004).

It has been known since the work of Urey (1947) and Bigeleisen and Mayer (1947) that temperature is crucial for the determination of equilibrium constants. These seminal works included calculations of equilibrium constants for isotopic exchange reactions as a function of temperature. At high temperature, the equilibrium constant becomes proportional to the inverse square of temperature (i.e., isotope fractionation decreases proportional to $1 / \mathrm{T}^{2}$ ). Thus, at the high temperatures involved in many Earth systems isotope fractionation was considered negligible and ignored for elements heavier than the traditional stable isotopes $(\mathrm{C}, \mathrm{H}, \mathrm{S}, \mathrm{O}, \mathrm{N})$. Similarly, the effect of pressure on isotope fractionation was also ignored.

Joy and Libby (1960) first calculated the effect of pressure on isotope fractionation. They suggested that oxygen isotope fractionation might be pressure dependent at low temperatures. However, in 1961, Hoering did not observe a pressure effect on oxygen isotope partitioning between water and bicarbonate at $43.5^{\circ} \mathrm{C}$ and 0.1 and $400 \mathrm{MPa}$. Then in 1975 , Clayton et al. found no pressure effect on the calcite-water fractionation at $500{ }^{\circ} \mathrm{C}, 0.1-2 \mathrm{GPa}$ and at $700{ }^{\circ} \mathrm{C}$, 50-100 MPa. Due to these initial studies, the effect of pressure on isotope fractionation was assumed to be negligible for all elements (except for hydrogen) at all pressures. However, in 1994, a study again predicted that pressure should have an effect on isotopic fractionation (Polyakov and Kharlashina 1994).

There are at least two ways in which pressure can affect isotope fractionation: differential molar volume decrease and force constant stiffening. The first cause is due to a molar volume isotope effect in which the heavy isotopes make slightly shorter bonds and therefore pack more tightly than lighter isotopes, and has been discussed in the literature extensively (e.g., Polyakov 1998). This effect comes mainly from the quantum vibrations of the nuclei inside their surrounding electronic cloud. The second cause is due to an increase of the force constants and, correspondingly, of the vibrational frequencies due to the stiffening of the bonds as the volume decreases due to increasing pressure.

The effect of oxygen fugacity on isotope fractionation was largely untested until the redoxactive transition elements and main group elements could be measured with adequate precision. Iron was the first element to be studied in great detail and since 1999 many studies have shown that, in both low and high temperature environments, the oxidation state $\left(\mathrm{Fe}^{3+}, \mathrm{Fe}^{2+}\right)$ exerts strong control on the magnitude of the Fe isotope fractionation (e.g., Williams et al. 2004; 
Shahar et al. 2008; Dauphas et al. 2014). Likewise, theoretical and experimental (e.g., Hill et al. 2009) results at low temperature have shown that speciation (bond partner and coordination in a solution) also regulates mass fractionation of Fe. In high-temperature solids or melts, the ligand to which Fe is bound also should affect the Fe isotopic fractionation (Schauble 2004). One of the main reasons that experiments are so critical to the study of equilibrium fractionation factors is that each of these variables can be studied individually in an experiment in order to determine which has the largest effect and how the variables influence each other. As mentioned above, Fe isotopes have been studied in the greatest detail experimentally, so many of the examples in this chapter will focus on Fe. However there are many elements that have been, or have the potential to be experimentally studied.

\section{PROOF OF EQUILIBRIUM IN ISOTOPE EXPERIMENTS}

The goal of typical stable isotope fractionation experiments is to determine the equilibrium fractionation factor between two phases at a set of temperatures, pressures, oxygen fugacities, and/or compositions of interest. The initial challenge for the experimentalist is to design the simplest, most elegant experiment that will achieve their goal, given the myriad constraints imposed by the laboratory. These issues include (but are not limited to) sample size, time, P-T capabilities of the experimental apparatus, availability and usefulness of appropriate starting materials and oxygen buffers, and the ability to separate phases for analysis after an experiment. For example, experimental apparati usually limit sample sizes to milli- or microgram levels, and realistic laboratory time scales impose challenges with systems involving diffusion as the method of isotope exchange. Additionally, and perhaps most importantly, is the ability to prove that the fractionation measured in an experiment is, in fact, the equilibrium fractionation factor between the phases of interest. This point is nontrivial and must be given significant thought when planning a set of experiments. This section will highlight some of the more effective experimental approaches and techniques employed in the past and speak to best practices for achieving a very close approach to equilibrium in stable isotope fractionation experiments.

\section{Time Series}

The use of a series of experiments conducted over a range of durations to assess the approach to equilibrium is one of the most commonly used methods in experimental petrology. The concept is straightforward: a series of experiments $(\geq 3)$ with the same starting materials is conducted at the same pressure and temperature (usually the lowest of interest) for increasingly longer durations. As the isotopes exchange between phases, the system moves closer to its equilibrium isotopic value with increasing experimental duration. The equilibrium fractionation between the two phases is estimated by running long enough experiments so that the system appears to have reached a steady state, i.e., there is no further detectable isotope exchange at progressively longer run times. The run products are then analyzed to ensure that they yield consistent results, which can be used to argue for a close approach to equilibrium (or at minimum a steady state). The time series results then guide future experiments.

A time series should also be used to assess chemical equilibrium independent of isotopic equilibrium. Chemical zoning in experimentally grown phases is always an indicator of disequilibrium and should lead to longer duration experiments whenever practical. An exception to this is dynamic cooling and/or decompression experiments designed to assess kinetic effects at controlled cooling and/or decompression rates. Some chemical zoning is tolerable in more 
traditional phase equilibrium experiments, as microbeam analyses can be made at phase boundaries where a local equilibrium may exist. However, most isotopic fractionation experiments require the physical separation of phases and analysis of the bulk phase. In this case chemical zoning is less tolerable as it will likely be accompanied by isotopic zoning. For this reason, the durations of phase equilibrium experiments designed to investigate isotopic fractionation may need to be longer than is typical for studies of element partitioning or the like (Shahar et al. 2008). In situ isotopic analyses using laser ablation MC-ICP-MS (LA-MC-ICPMS) or secondary ion mass spectrometry (SIMS) is a way to determine if isotopic zoning has occurred in an experiment, and to obtain spatially oriented measurements of the experimental product. However, extreme care must be taken when treating the resulting data to show the measured values represent a close approach to equilibrium.

\section{Multi-Direction Approach}

The multi-direction approach is also discussed in O'Neil (1986) and has been termed "reverse reactions" in the experimental petrology community (Fig. 1). It is an extension of the time series described above with the added benefit of approaching the equilibrium isotope value from two sides, which results in a more precise determination of the equilibrium value by bracketing. This is done by conducting at least two time series in which the difference between the isotopic compositions of the starting materials lie on either side of the equilibrium isotope fractionation value of the system (line labeled 'Equilibrium' in Fig. 1). Over time, the two sets of starting materials (differing only in isotopic value) evolve towards the equilibrium isotopic state of the system, thereby bracketing the true equilibrium fractionation factor of the two phases. Although this idea seems straightforward there are subtleties associated with non-ideal conditions and solid phases that could complicate the system which are discussed in great detail by Pattison (1994). Although the multi-direction approach is more rigorous than a simple time series, its accuracy in determining the equilibrium fractionation factor is limited by the ability to come close to equilibrium in laboratory time scales. For some systems, this technique may not be practical due to sluggish isotope exchange. In such cases, a different strategy is required to determine equilibrium fractionation factors.

The Northrop-Clayton method is a variation of the multi-direction approach that allows an estimation of the equilibrium fractionation between two phases by extrapolation. This is a strategy that can be employed when attaining equilibrium is not possible in time scales appropriate for laboratory experiments. This approach, termed the "partial exchange technique" in O'Neil (1986), was first described by Northrop and Clayton (1966) and later by Deines and Eggler (2009). The partial exchange method involves two phases that each contain at least one site for an element of interest that exchanges isotopes throughout an experiment. A series of experiments are conducted with identical solids and several isotopically variable fluids that bracket the equilibrium isotopic fractionation factor. Just like in the multi-direction approach, the evolution of the fractionation with time will bracket the equilibrium value.

The benefit of the Northrop-Clayton method is that a close approach to equilibrium is not required to estimate the fractionation factor. This is true because this method assumes that rates of isotopic exchange for 'companion' experiments (runs that are the same in every respect except the isotopic composition of starting materials) are identical. This assumption allows one to extrapolate to the equilibrium value of the system based on the 'percentage of isotopic exchange' in two or more companion experiments (O'Neil 1986). For this method, the extent of isotopic exchange is directly proportional to the accuracy of the fractionation factor. In other words, if 
the isotopes are not largely exchanged, the fractionation might appear to be larger than the true equilibrium value. A combination of this partial exchange technique and the classical time series approach was used successfully by Schuessler et al. (2007) in obtaining the equilibrium Fe isotope fractionation between pyrrhotite and melt.

\section{Three-Isotope Technique}

Another method of determining equilibrium fractionation factors is termed the three-isotope exchange method. This method was pioneered at the University of Chicago (Matsuhisa et al. 1978; Matthews et al. 1983) for oxygen isotopes between a mineral and an aqueous phase and later modified by Shahar et al. (2008) to determine direct mineral-mineral fractionation of Fe isotopes. This method requires that the element of interest has at least three stable isotopes in measureable abundances and utilizes the addition of a known amount of isotopic 'spike' (addition of a specific isotope to a phase in excess of its natural abundance) to one of the starting materials containing the element of interest. The choice of which isotope to use as the spike is made obvious by looking at a three-isotope plot (Fig. 2). By adding an excess amount of the isotope present in the denominator of both axes (represented by an ' $x$ ' in the axes labels on Fig. 2A, B) to one of the starting materials, the spiked phase will be displaced from the terrestrial fractionation line (TFL) in three-isotope space by a line with a slope of unity.

The principle of the three-isotope exchange method is to replace the TFL, which has a zero intercept on a three-isotope plot, with a secondary fractionation line (SFL) with the same slope as the TFL, but a non-zero intercept determined by the bulk composition of the isotopically spiked system. Figure $2 \mathrm{~A}$ depicts the typical trend to equilibrium expected in these experiments. As the spiked (Phase 2 Initial) and unspiked (Phase 1 Initial) starting materials exchange isotopes at the pressure and temperature of interest for increasingly long times $\left(t_{1}, t_{2}\right)$, their isotopic compositions migrate towards the SFL. When isotope exchange is complete and the system reaches isotopic equilibrium, all phases containing the element of interest will lie on the SFL (Phase 1 \& 2 Equilibrium), and the equilibrium fractionation factor will be defined by the distance between the final measured values along the $\mathrm{x}$ - and $\mathrm{y}$-axes corresponding to isotopes ' $\mathrm{j}$ ' and ' $i$ ', respectively. The three-isotope exchange method can also be used to extrapolate to the equilibrium isotopic value in cases where exchange is slow. In this case it is quite important that the experiment not be 'over' spiked so as to limit the amount of extrapolation that is necessary. A good rule of thumb is to incorporate no more (and sometimes much less) than $1 \%$ of the element as a spike.

By way of practical example, we turn to the three Fe isotopes, a system for which the threeisotope method has been executed successfully for mineral-mineral, mineral-fluid, and metalsilicate exchange experiments (e.g., Shahar et al. 2008; Beard et al. 2010; Shahar et al. 2015; Elardo and Shahar 2016). For Fe, the axes of the three-isotope plot are defined by two isotope ratios with the same denominator: ${ }^{56} \mathrm{Fe} /{ }^{54} \mathrm{Fe}$ on the y-axis and ${ }^{57} \mathrm{Fe} /{ }^{54} \mathrm{Fe}$ on the $\mathrm{x}$-axis (see Fig. 2 of Shahar et al. 2008). These ratios are compared to those of a standard and reported in per mil (\%) using the conventional delta notation:

$$
\delta^{i} \mathrm{Fe}=10^{3}\left(\frac{\left({ }^{i} \mathrm{Fe} /{ }^{54} \mathrm{Fe}\right)_{\text {Sample }}}{\left({ }^{i} \mathrm{Fe} /{ }^{54} \mathrm{Fe}\right)_{\text {Standard }}}-1\right)
$$


where $i=56$ or 57 . If the goal is to derive the equilibrium isotope distribution by extrapolation, one can take advantage of the known equilibrium mass fractionation relationship between the two isotopes of interest:

$$
\delta^{56} \mathrm{Fe}=\left(10^{3}+\delta^{56} \mathrm{Fe}_{\text {bulk }}\right)\left(\frac{10^{3}+\delta^{57} \mathrm{Fe}}{10^{3}+\delta^{57} \mathrm{Fe}_{\text {bulk }}}\right)^{\gamma}-10^{3}
$$

This slightly concave fractionation relationship can be approximated by a straight line in threeisotope space with a slope of approximately $\gamma$ and an intercept defined by the bulk isotopic composition. The intersections of the line defined by the above equation for the SFL and the two lines derived from the trajectories of the isotopically evolving starting materials represent extrapolation to the equilibrium values of the system. The exponent, $\gamma$, is 0.67795 (for $\mathrm{Fe}$ ) at equilibrium based on the equation for equilibrium mass fractionation:

$$
\gamma=\frac{\frac{1}{m_{56}}-\frac{1}{m_{54}}}{\frac{1}{m_{57}}-\frac{1}{m_{54}},}
$$

where $m$ is the mass of subscripted Fe isotope. Although both methods offer extrapolation to equilibrium, an advantage of the three-isotope technique over the Northrop-Clayton method is the ability to know when isotopic exchange is complete and equilibrium has been reached, i.e., when the final isotope values lie on the SFL.

An entirely different path towards equilibrium using the three-exchange isotope method is presented schematically in Figure 2B. Here, the spiked (Phase 2 Initial, $\mathrm{t}_{0}$ ) and unspiked (Phase 1 Initial, $t_{0}$ ) starting materials are thoroughly chemically and isotopically mixed before equilibrating because either the sample is completely melted or dissolved during the experiment. When the system homogenizes at the beginning of the experiment $\left(\mathrm{t}_{1}\right)$, the isotopes mix and collapse to the bulk isotopic value of the system (yellow star), which by definition lies on the secondary fractionation line (SFL). Following mixing $\left(t_{1}\right)$, there is an 'unmixing' $\left(t_{2}, t_{3}\right)$ among the isotopes along the secondary fractionation line (SFL) as the system equilibrates isotopically as well as chemically over time. In progressively longer experiments, the isotopic values of the phases move away from each other along the SFL until they reach their equilibrium values (Phase 1 \& 2 Equilibrium, $t_{4}$ ). In this case, equilibrium is determined by time series during unmixing; i.e., when the values stop changing (reach a steady state) along the SFL, the system is thought to be in equilibrium and the fractionation factors can be determined. This is critically important and considered a rigorous way to prove equilibrium in these experiments.

The utility of the three-isotope method is in the ability to trace the trajectories of isotopic exchange between phases in three-isotope space as the experiments progress through a time series. Without an isotope tracer (the isotopic spike) it could be difficult to prove that isotopic equilibrium had been reached, know whether the different phases had exchanged isotopes, and be certain that the exchange occurred in a closed system. By spiking one of the phases in the experiments with a known amount of spike, there is better control on mass balance and one can determine if the experiment was truly a closed system. Open system behavior was successfully diagnosed by Lazar et al. (2012) in three-isotope experiments involving $\mathrm{Ni}$ alloying with $\mathrm{Au}$ capsules. 
Proving that the isotopes have come to equilibrium is the most crucial part of conducting the high temperature experiments. If the isotopes are mixed but have not equilibrated yet then the fractionation would either be underestimated or determined as zero. In silicate-metal experiments, the majority of the $\mathrm{Fe}$ in the experiment is in the metallic phase, so any contamination from the metal to the silicate (which cannot be seen by the eye) will cause the apparent fractionation factor to underestimated or appear to be zero. However, with the threeisotope exchange method this can be easily seen and a duplicate experiment would be performed and more carefully separated to obtain the correct result. Also, it is important to remember that chemical equilibrium is not equivalent to isotopic equilibrium and must be independently assessed. Shahar et al. (2008) found that while chemical equilibrium between fayalite and magnetite occurred within 6 hours, isotopic equilibrium required 48 hours at $800^{\circ} \mathrm{C}$. This is extremely important for experiments where equilibrium is assumed to have occurred based on electron probe measurements showing equilibrium textures and chemical compositions.

The use of a classical time series to assess the approach to equilibrium in isotopic fractionation experiments is valuable even in conjunction with other methods and we highly recommend its use in all experimental studies of isotope fractionation. Furthermore, the threeisotope exchange method may not be available for use in all systems of interest. In such cases, the use of a time series is essential and is highly recommended in conjunction with a multidirectional approach. Frierdich et al. (2014) argued that even the three-isotope technique is not sufficient on its own to guarantee that there are no possible kinetic uncertainties in the data. The authors argued that adding a multi-direction approach to the three-isotope technique unambiguously demonstrates that equilibrium has been reached during the experiment. Investigators should thoughtfully develop an experimental plan involving some combination of the techniques presented above that will most rigorously yield the equilibrium isotope fractionation factors sought for their system.

\section{Kinetic Effects}

Kinetic isotope effects are commonly observed in nature and in the lab, making it crucial to be able to distinguish between isotopic equilibrium and disequilibrium. Both states are important to understand and quantify for isotopic systems, but the experimentalist must be able to discern which of the two is represented in their experiments. Kinetic isotope effects are usually associated with fast, unidirectional, or incomplete processes (O'Neil 1986). Some of the pathways that might lead to kinetic effects in isotope exchange experiments are evaporation, diffusion, and rapid crystallization. No matter how carefully an experiment is designed there is always the possibility of a kinetic isotope effect that has not been considered. From quenching effects in a piston cylinder to isotopic zoning within crystals, it is nearly impossible to avoid all kinetic isotope effects. However, this does not mean that the equilibrium value cannot be determined if the kinetic processes are properly identified and understood, and the data are treated accordingly. For example, Shahar et al. (2011) noted that when measuring Si isotopes in situ by laser ablation MC-ICP-MS, the isotopic values within each phase showed small deviations thought to be due to a kinetic effect during the quenching of the experiment. In order to determine the true isotopic ratio of the phases, the values were averaged. Had the experiment been measured by solution instead of by laser ablation these local variations would not have been seen and the kinetic behavior would have gone unnoticed.

In some cases kinetic processes are not an impedance to equilibrium, but a means to achieve it. For example, diffusion may be the dominant mechanism by which isotopes exchange between 
phases in an experiment. In this case, it is important to understand the rates of diffusion corresponding to the isotopes and materials of interest. Diffusion coefficients for many systems have been experimentally determined and can be used in some cases to make predictions as to how long it should take for two phases to chemically and isotopically equilibrate at the pressure and temperature of interest. This information can be used to guide the experimentalist when deciding on experimental durations in a time series.

In an experiment depending on diffusion (or another kinetic process) to exchange isotopes, it is critically important to allow enough time for a steady state to be reached so that a reasonable estimation of equilibrium can be made. However, it is also possible to achieve a spurious result by running an experiment for too long. For example, Lazar et al. (2012) suggested that there is a divergence in the isotope ratios after an experiment had reached equilibrium when kinetic effects can start to play a role again, especially when there is diffusive loss to the capsule. Therefore it is important to remember that while a time series is a good way to prove that isotopic equilibrium has been reached, there is a limit to the duration of the experiment that should not be passed. These are just two of many examples of how kinetic effects can be found within experimental charges even when isotopic equilibrium has been reached.

\section{EXPERIMENTAL METHODS}

Since the advent of MC-ICP-MS and associated chemical purification methods that allow for the accurate measurement of small isotopic fractionations between co-existing materials, researchers have been exploring pressure, temperature, and composition space in non-traditional stable isotope exchange experiments. Drawing from the well-established experimental methods used successfully by geochemists and petrologists for decades, as well as newer methods developed by mineral physicists and beam line scientists, the community is making great advances in our understanding of how non-traditional stable isotopes fractionate in geo- and biogeochemical processes. In order to reproduce the range of conditions occurring from Earth's surface to its core, researchers are utilizing a variety of experimental apparati, including simple flasks and low-temperature ovens, cold-seal hydrothermal vessels, piston-cylinders, multi-anvils, and diamond anvil cells. In the following sections we provide a brief (non-exhaustive) review of these methods, starting with low temperature experiments and their applications, and then moving progressively higher in temperature and pressure.

\section{Low Temperature Experiments}

Early experiments on the non-traditional stable isotopes focused on low temperature Fe isotope fractionation in aqueous fluids (e.g., Johnson et al. 2002; Welch et al. 2003; Hill et al. 2009) and more commonly between an aqueous fluid and a mineral (e.g., Johnson et al. 2002; Skulan et al. 2002). At this time in Fe isotope geochemistry history, it was unclear if abiotic systems could fractionate isotopes as efficiently as biologic systems, so a baseline was needed in order to determine if $\mathrm{Fe}$ isotopes could be used as a biosignature. These experiments employed the use of an isotopic spike (excess ${ }^{57} \mathrm{Fe}$ ) in combination with classical time series and multidirection approaches to assess Fe isotope fractionation between phases at low temperatures. Johnson et al. (2002) and Welch et al. (2003) investigated fractionation in aqueous Fe(II) and $\mathrm{Fe}$ (III) solutions at room temperature and in an ice bath. These studies have implications for the fractionation of $\mathrm{Fe}$ isotopes between aquo and hydroxy complexes in oxygenated natural waters.

If isotope fractionation associated with a redox change exists it would be most noticeable at 
low temperature and amongst two phases with strikingly different bonding environments, such as a fluid and a solid. As explained in detail in Skulan et al. (2002), low-temperature mineral-fluid exchange experiments rely on a steady state of dissolution and precipitation so that the isotopes can move around within the experiment and tend towards equilibration. This study utilized a range of experimental strategies to ultimately distinguish kinetic effects from equilibrium fractionation between hematite and aqueous $\mathrm{Fe}(\mathrm{III})$. One approach they used was simply mineral synthesis. The premise is that a mineral, such as hematite, will be thermodynamically stable at the conditions of the experiment, but will not be present in the starting materials, thus facilitating precipitation. In this case, Skulan et al. (2002) reacted aqueous $\mathrm{Fe}$ (III) with dilute $\mathrm{HNO}_{3}$ in sealed Pyrex flasks at $98{ }^{\circ} \mathrm{C}$ to precipitate hematite from solution. The first hematite crystals will not be in isotopic equilibrium, but as the experiment progresses the system will reach a steady state of dissolution and precipitation in which the hematite crystals will then record the equilibrium fractionation between the crystal and the fluid.

The main disadvantage to these experiments is that at low temperature it is much more difficult to attain isotopic equilibrium as the rates of diffusion and nucleation are slow. There are also several kinetic effects that must be sorted out such as isotopic inhomogeneity in the mineral (i.e., zoning during precipitation) or other kinetic effects associated with the rates of dissolution and precipitation. In order to speed up diffusion rates, hydrothermal experiments can also be done using a cold seal technique at slightly elevated temperatures. For example, Li et al. (2015) determined the equilibrium $\mathrm{Mg}$ isotope fractionation between dolomite and aqueous $\mathrm{Mg}$ in multidirection mineral synthesis experiments using a ${ }^{25} \mathrm{Mg}$ spike to track isotope exchange. These experiments were conducted at $130-220^{\circ} \mathrm{C}$ using Parr bombs and an internal sealed container. By using this technique the experimentalist can avoid possible kinetic effects due to energy barriers that could inhibit crystal growth or dissolution.

Another subject of interest in low temperature experimental work is the isotopic effect of adsorption onto mineral surfaces (e.g., Barling and Anbar 2004; Icopini et al. 2004; Johnson et al. 2004; Beard et al. 2010; Nakada et al. 2013; Wasylenki et al. 2014; 2015). These experiments are aimed at understanding the isotope fractionation between aqueous metal species and sorbed metal complexes. In many of these experiments it has been shown that the isotope fractionation is constant throughout the experiment even as the amount of the element sorbed increases. To prove isotopic equilibrium in these experiments a time series and/or the three-isotope technique has been used; we recommend the use of both whenever possible. Additionally, in these types of experiments, the fractionation of interest in nature might be due to kinetic processes or opensystem equilibrium-driven fractionation. Therefore, the approach to treating and interpreting the data may include modeling by constant offset as a function of reactant/product ratio (equilibrium, closed-system), or as Rayleigh behavior (equilibrium open-system, or kinetic). For a more thorough explanation of these different treatments, see Johnson et al. (2004)

\section{High Temperature, Low Pressure Experiments}

The least amount of experimental work to date has focused on experiments at hightemperature and ambient or low pressure $(\leq 0.5 \mathrm{GPa})$. This is due in part to the assumption that pressure was not an important parameter in isotope fractionation, and the more practical concern that it is typically easier to achieve a closed system when conducting high-pressure experiments. However, there have been a few key studies conducted in low-pressure experimental apparati in order to study the effect of oxygen fugacity and temperature on isotope fractionation. As far as we are aware, the first experiment on fractionation between a silicate melt and metal was 
conducted in this fashion in a 1 atm gas-mixing furnace (Roskosz et al. 2006). Figure $3 \mathrm{~A}$ depicts a cutaway of the interior of such a furnace, exposing a molten sample suspended by a metal wire loop (e.g., $\mathrm{Pt}, \mathrm{Re}$ ) in a sealed alumina or mullite tube, which is flushed with an appropriate gas mixture (e.g., $\mathrm{CO}-\mathrm{CO}_{2}, \mathrm{CO} 2-\mathrm{H}_{2}$ ) to impose the chosen oxygen fugacity.

In the study by Roskosz et al. (2006), it was shown that at $1500^{\circ} \mathrm{C}$ there was a resolvable and quite large fractionation of Fe isotopes between silicate melt and metal in a series of Pt loop experiments, although it proved difficult to demonstrate isotopic equilibrium. These experiments used the propensity for $\mathrm{Fe}$ to alloy with $\mathrm{Pt}$ as a way to segregate the metal from the silicate at a fixed $f \mathrm{O}_{2}$ and temperature. The authors noted that kinetic fractionation was rampant during the first $~ 300$ minutes of an experiment. Initially, the Fe alloying with the Pt was isotopically light compared to that in the silicate melt and became heavier as the experiment approached equilibrium. In the longest duration experiment, the relative fractionation flipped such that the silicate was lighter than the metal, thus illustrating the need for, and utility of, conducting a time series in such experiments.

Another low-pressure study by Schuessler et al. (2007) used an internally heated pressure vessel (IHPV) to investigate Fe isotope fractionation between a rhyolitic melt and pyrrhotite between $840{ }^{\circ} \mathrm{C}$ and $1000{ }^{\circ} \mathrm{C}$ at $0.5 \mathrm{GPa}$. A rigorous assessment of the approach to equilibrium was employed in this study. Three sets of experiments were done to distinguish kinetic effects from equilibrium fractionation for the system. The first was a classical time series in which ${ }^{57} \mathrm{Fe}$ spiked glass was reacted with natural pyrrhotite to determine the time required for a close approach to equilibrium. The second set of experiments employed the Northrop-Clayton method of partial isotope exchange discussed above to extrapolate to the equilibrium value (Northrop and Clayton 1966). In the third set, pyrrhotite was crystallized from the melt instead of being present in the starting materials. This combination of experimental approaches essentially constituted a multi-direction assessment of equilibrium supported by time series.

The studies of Roskosz et al. (2006) and Schuessler et al. (2007) demonstrated the utility of controlled-atmosphere furnaces and IHPVs for isotope fractionation experiments, but challenges in technique development remain. Although the controlled-atmosphere furnace is an ideal tool for controlling the oxygen fugacity, an important variable for isotope studies that is more difficult to control precisely in high pressure experiments, further work will need to assess whether isotope systems of interest remain in a closed system so that equilibrium is more rigorously demonstrated. If an open system exists with respect to the isotopes, kinetic isotope effects will dominate. It is best practice for investigators studying isotope fractionation in a controlled-atmosphere furnace to conduct a time series with as few phases as possible (e.g., a time series of super-liquidus experiments) to assess whether the bulk system retains a constant bulk isotopic composition before attempting phase equilibrium experiments where open system behavior may obscure or be difficult to disentangle from additional kinetic or equilibrium fractionation between phases.

Other challenges for low-pressure experiments may include (but not limited to) the effects of slow diffusion creating chemical and isotopic zoning in solid mineral or metal phases and difficulty in obtaining pure phase separates post-experiment. We recommend that the interested investigator draw upon the extensive literature regarding the use of experimental techniques such as controlled-cooling rates (e.g., Donaldson 1976; Corrigan 1982), temperature cycling (e.g., Mills and Glazner 2013), and crystal seeding (e.g., Larsen 2005; Dalou et al. 2012) to develop methodology to overcome these issues. Further isotope work using a controlled-atmosphere furnace or IHPV will need to build on the pioneering work of Roskosz et al. (2006) and 
Schuessler et al. (2007), as these types of experiments potentially have huge advantages over high pressure experiments, such as long-term temperature stability, large sample masses, and precise control over oxygen fugacity.

\section{High Temperature and Pressure Experiments}

Many experiments to date have been carried out in a piston cylinder apparatus (Fig. 3B) and a few in the multi-anvil apparatus (Fig. 3C). Both of these instruments allow the experimental materials to stay in a sealed container while at the pressure and temperature of interest throughout the experiment. The benefit is that at elevated pressures and temperatures, equilibrium is more easily attained, and with closed capsules, the composition is less likely to change throughout the experiment. Most experiments are carried out using a $1 / 2$-inch pistoncylinder cell assembly (Fig. 3B), which is typically capable of maintaining pressures up to $\sim 4$ $\mathrm{GPa}$. The cell assembly varies by laboratory but a typical assembly, as illustrated by Figure $3 \mathrm{~B}$, consists of a graphite tube heater surrounded by a pressure medium, the most common of which are $\mathrm{BaCO}_{3}$, talc or $\mathrm{NaCl}$ with an inner Pyrex sleeve, and occasionally $\mathrm{CaF}_{2}$. A thermocouple is inserted axially into the cell through a small hole in the stainless steel base plug, which is typically surrounded by a thin insulating pyrophyllite or Pyrex sleeve. Capsule material also varies according to the specific needs of the experiment (e.g., graphite, $\mathrm{Au}, \mathrm{Pt}$ ), and capsules are typically centered within the cell. Hotspots are typically large (i.e., on the order of millimeters) in piston-cylinder experiments, so the thermal gradient over a sample is not expected to exceed $\sim 15{ }^{\circ} \mathrm{C}$ in most cases. The first experiment to use the piston cylinder apparatus to directly determine mineral-mineral fractionation was a precipitation-dissolution experiment to measure the Fe isotope fractionation between fayalite and magnetite (Shahar et al. 2008).

Multi-anvil cells have smaller sample sizes but can extend the accessible pressure range to approximately $25 \mathrm{GPa}$ (Fig. 3C). Multi-anvil assemblies typically consist of a ceramic octahedron with a cylindrical sample chamber drilled through the center. A heater, typically made of graphite, rhenium-foil, or lanthanum chromite surrounds the sample capsule and confining spacers. A thermocouple can be inserted either axially or radially depending on the assembly. Although the multi-anvil apparatus can access a far greater pressure range than a piston cylinder, there is a significant tradeoff with sample volume. The maximum pressure achievable by a particular multi-anvil cell assembly is inversely proportional to the maximum sample size within that assembly. For an in-depth explanation and visualization of pistoncylinder and multi-anvil experimental setups, the interested reader is referred to Bennett et al. (2015).

One of the most important choices an experimentalist has to make is what type of capsule to use in an experiment. The choice of capsule material is crucial to the success of the experiment, as in order to assess the approach to isotopic equilibrium and ensure that the possibility of kinetic processes is limited, a closed system must be maintained. An open system, with respect to isotopes, will in all likelihood result in non-equilibrium conditions during the experiment. Therefore, a capsule that alloys or reacts with the element of interest should not be used for the experiment (e.g., metal capsules in metal-silicate experiments simulating core formation). This would lead to a loss of the element of interest into the capsule or the creation of a mineral on the capsule wall that has an isotope fractionation of its own (possibly kinetic) and would therefore change the mass balance of the system and the fractionation throughout the experiment.

There is no perfect capsule material in experimental petrology, but acceptable choices can be found. Graphite capsules are the most popular choice. Graphite acts as an inert phase in most 
systems and is well-suited for many investigations, provided that the condition of carbon saturation can be tolerated. Noble metal and alloy capsules are another popular choice. Platinum, $\mathrm{Re}, \mathrm{Au}, \mathrm{Ag}$, and Au-Pd are the most commonly used metal capsules; each having its own strengths and drawbacks. The most popular metal capsule, Pt, will cause Fe depletion from silicate melts (Merrill and Wyllie 1973). Although this behavior is well-characterized in the literature and such capsules can be pre-saturated with Fe to minimize this issue, the Pt will provide an additional "sink" for Fe that must come to isotopic equilibrium with the melt in order to avoid kinetic fractionations. Other potential capsule materials include olivine, magnesia, alumina, boron nitride, and silica glass/fused quartz. When the parameters of an experiment require a compromise on capsule material, it is best to test the conditions of interest with more than one capsule material to confirm the results.

Isotope fractionation experiments present an additional challenge over more traditional high P-T experiments in that two phases of interest typically need to be physically separated and analyzed in solution by MC-ICP-MS. As the achievable pressure range of an experimental apparatus increases, sample sizes necessarily decrease and the ability of a researcher to truly separate the phases, purify the element of interest, and obtain a precise isotope ratio becomes more limited. A potential work around is to use a selective leaching process (Williams et al. 2012) to chemically separate the phases of interest. However, the possibility of isotopic fractionation during the leaching process exists. For this reason though there are not as many published studies of multi-anvil experiments as piston cylinder experiments. However, it is expected that more researchers will push the current boundaries with this technique, especially if using a large volume multi-anvil press.

Selective leaching (also called preferential dissolution) as a strategy to separate phases postexperiment was also used successfully by Macris et al. (2013). This study determined the equilibrium $\mathrm{Mg}$ isotope fractionation between spinel and forsterite in piston-cylinder experiments at $1 \mathrm{GPa}$ and $600-800{ }^{\circ} \mathrm{C}$. Two Au capsules were placed side by side in the cell assembly, one containing spinel spiked with excess ${ }^{24} \mathrm{Mg}$ and 'normal' magnesite, and the other containing forsterite spiked with excess ${ }^{24} \mathrm{Mg}$ and 'normal' magnesite. The three-isotope technique was used to determine the spinel-magnesite fractionation and the forsterite-magnesite fractionation, then the spinel-forsterite fractionation was obtained by difference. The magnesite in these experiments served two purposes: it acted as the $\mathrm{Mg}$ isotope exchange medium and it allowed for complete separation of phases after the experiment by preferential dissolution. The use of carbonate as an exchange medium has been well known since the pioneering oxygen isotope fractionation experiments of Clayton et al. (1989) and Chiba et al. (1989). It is thought to be superior to water as an isotopic exchange medium and reference material because of potential 'isotope salt effects' that can affect the observed fractionation in an experiment due to mineral dissolution changing the fluid composition (Hu and Clayton 2003).

Experiments in the piston cylinder have relied on a time series approach or utilized the threeisotope technique to address isotopic equilibrium. Whenever possible both methods should be used to demonstrate a close approach to equilibrium. The Fe isotope system has been the mostwell studied system thus far with the piston cylinder (Poitrasson et al. 2009; Hin et al. 2012; Shahar et al. 2015; Elardo and Shahar 2016). However the piston cylinder has proven useful for studies of many other isotope systems such as S (Labidi et al. 2016), Mo (Hin et al. 2013), Cr (Bonnand et al. 2016), Si (Shahar et al. 2009; Kempl et al. 2013; Hin et al. 2014), Mg (Richter et al. 2008; Huang et al. 2009; Macris et al. 2013), Ni (Lazar et al. 2012) and more. Multi-anvil experiments have been conducted mostly for Fe isotopes (Poitrasson et al. 2009; Williams et al. 
2012), but also for Si isotopes (Shahar et al. 2011). It is our belief that these experiments will begin to become more commonplace, leading to a more complete database of experimentally determined equilibrium fractionation factors.

\section{NRIXS and Diamond Anvil Cell Experiments}

One might imagine that if multi-anvil experiments have sample sizes that are almost too small to be useful, then diamond anvil cell experiments would be all but impossible. However, it is important to know if pressure truly is a negligible variable within isotope geochemistry (e.g., Clayton et al. 1975). Therefore, a new technique has emerged in order to measure isotope fractionation factors at high pressure. Polyakov and co-workers (Polyakov et al. 2005; 2007; Polyakov 2009) have pioneered the use of nuclear resonant inelastic X-ray scattering (NRIXS) synchrotron data to obtain vibrational properties of minerals from which isotopic fractionation factors can be calculated. The technique is based on the fact that certain isotopes possess a lowlying nuclear excited state that can be populated by X-ray photons of a particular energy and therefore directly probe the vibrational properties of the solid.

As shown in Figure 3D, in nuclear resonant X-ray spectroscopy an in-line high-resolution monochromator is used to narrow down the photon energy to $\mathrm{meV}$ resolution and fine-tune the monochromatic X-ray near the exceedingly narrow nuclear resonant (elastic) line. Avalanche photodiodes (APD) are used to collect only the signal from nuclear resonance absorption and reject non-resonantly scattered radiation. The APD directly downstream of the sample collects nuclear resonant forward scattering, or synchrotron Mössbauer spectroscopy (SMS) signal, which can provide a precise determination of the hyperfine interaction parameter and LambMössbauer factor. The APDs surrounding the sample radially collect the NRIXS signal, which is a result of creation (Stokes) or annihilation (anti-Stokes) of phonons as the incident X-ray beam is scanned over a small range (approximately $\pm 100 \mathrm{meV}$ ) around the resonant energy. The phonon density of states (DOS) is extracted from this phonon excitation spectrum associated with the nuclear resonant isotope (Hu et al. 2003). The integration of the whole DOS provides values for key thermodynamic properties. In addition, NRIXS data can also be used to derive reduced partition function ratios ( $\beta$-factors) from which equilibrium isotopic fractionation factors can be calculated: $\delta_{\mathrm{A}}-\delta_{\mathrm{B}}=1000 *\left(\ln \beta_{\mathrm{A}}-\ln \beta_{\mathrm{B}}\right)$, where $\mathrm{A}$ and $\mathrm{B}$ are two different phases of interest. This technique has been widely used by the mineral physics community to investigate seismic velocities and phonon density of states of high-pressure minerals (Sturhahn et al. 1995) but is relatively new to the isotope geochemistry community.

The most commonly used software for processing NRIXS data is the PHOENIX program. The PHOENIX program (Sturhahn 2000) analyzes the data obtained by NRIXS and exports three different force constants. The first is the determination of the force constant using the moments approach in which the normalized excitation probability is computed using the raw data and the experimental resolution function. The outcome is based on the raw data and therefore multiphonon contributions are included in the analysis. The second determination of the force constant is also based on this moments approach; however it is based on data that are first 'refined,' that is, the multiphonon contribution is corrected for and $\mathrm{S}(\mathrm{E})$ is extrapolated. Dauphas et al. (2012) argued that this moments approach is the best method for determining the force constant as it has smaller uncertainties, is not as dependent on the background, and is not as sensitive to asymmetric scans. The third determination of the force constant is based on the partial density of states that is computed from the raw data. The force constant is then calculated based on the density of states and is therefore only based on the one phonon contribution of the 
data and does not need to be corrected for the possible multiple phonon contributions in the data. Murphy et al. (2013) suggested that this last force constant calculation is the most robust. The two methods produce slightly different force constants thereby changing the beta factor calculation as well.

Dauphas et al. (2012) also suggested that the high-pressure data used by Polyakov (2009) were inaccurate due to the truncation of the high-energy tail during the data acquisition. These data were originally collected by different groups to derive seismic velocities and were not specifically intended for the novel applications pioneered by Polyakov (2009) regarding $\beta$ factors. However, since this type of data has proven effective in calculating equilibrium isotope fractionation factors, its collection and treatment has been improved. For example, Dauphas et al. (2012) further suggested that longer acquisition times are necessary to determine the partial phonon density of states (PDOS) of the phases at high pressure with particular emphasis on the high energy tail. To aid in this, Dauphas et al. (2014) introduced a new program, SciPhon, which determines the background on each side of the elastic peak and subtracts them individually instead of using one background value for the whole spectral range. The broadest spectral energy ranges allow for the most accurate determination of the background correction, apparently reducing overall uncertainty. A comparison of the force constant obtained from PHOENIX and SciPhon indicate that SciPhon uses more accurate background subtraction, gives values closer to theoretical calculations, and is more consistent over differing energy ranges (Shahar et al. 2016). Therefore, SciPhon should be used to calculate force constants when conducting NRIXS experiments. Roskosz et al. (2015) successfully applied SciPhon to NRIXS data to describe the equilibrium Fe isotope fractionation between spinels and silicates, which is in agreement with the direct experimental determination by Shahar et al. (2008).

Once the force constant is calculated, the $\beta$-factor determination is straightforward (e.g., Polyakov 1998; Murphy et al. 2013). These calculations are then used to obtain an estimate of the equilibrium isotope fractionation as described above: the $\beta$-factors are calculated for each phase that is being probed and then subtracted from one another to get the isotopic fractionation between the two phases. In this way, each phase is analyzed in situ and does not need to be separated post-experiment. To date, this technique has been used solely for room temperature experiments, both at atmospheric pressure and high pressure (Dauphas et al. 2012; Shahar et al. 2016). It is expected that high temperature experiments will follow when the temperature gradients in the diamond anvil cell can be better controlled and reduce uncertainties in the analyses.

\section{POST-EXPERIMENT ANALYSIS}

The characterization of run products for experiments designed to investigate isotopic fractionations is typically more in-depth than for traditional petrologic experiments, and in most instances requires more sample mass. For low-temperature experiments, both the phases involved and experimental techniques employed are more varied, and are often specific to the system being investigated. Here we will focus on the process for high-temperature experiments by way of example.

Ambient-pressure, piston-cylinder, and multi-anvil experimental run products are all similar in their analytical requirements. Once the samples have been removed from the experimental

assembly, the samples are broken or cut in half. For piston-cylinder and especially multi-anvil assemblies, it is best practice to cut the sample capsules axially rather than radially in order to 
assess any compositional gradients that may be present due to temperature gradients in the cell. The first portion of the run product is mounted and polished for electron microprobe analysis. Geochemical characterization of the run product via electron microbeam analyses is essential and should always be conducted in tandem with isotopic analyses.

The rest of the sample is then processed for isotopic analyses. The most effective method for phase separation will likely vary with the phases of interest. Fortunately, in the case of experiments simulating the process of core - mantle differentiation, Fe metal alloys and quenched silicate melt have extremely different visual properties and are often easily separated by gently crushing the run product, followed by hand picking separates with the aid of a binocular microscope. The metal phase is typically very easy to mechanically separate, whereas hand picking a pure quenched silicate melt phase is more difficult. The portions of sample that appear to be pure are then placed under a strong magnet and screened thoroughly to ensure that the silicate portions are not at all magnetic. Only the silicate portions that make it through that test are dissolved in strong acids for isotopic analyses. After acid-digestion, the element of interest is chemically separated and purified by column chemistry for isotopic analyses, usually by MC-ICP-MS. Chemical purification procedures will vary for different isotopic systems and phases of interest, as will the analytical instrumentation and measurement procedures. Reviewing these procedures is beyond the scope of this chapter; however excellent reviews for isotopic systems of interest are available in the following chapters of this volume.

These post-experiment steps (phase separation and dissolution) are not necessary if the isotopic analyses are conducted in situ by LA-MC-ICP-MS. In this case, the second portion of the sample capsule will be mounted in epoxy and polished prior to laser ablation. To our knowledge, no study has yet physically separated quenched silicate melt from a mineral phase of interest to measure the isotopic fractionation. Ensuring a "clean" mineral phase may prove difficult for experimental run products of this kind. The crystallization kinetics during the experiment will undoubtedly prove important in growing crystals that are both free of melt inclusions or embayments and large enough to mechanically separate from the quenched melt. In cases such as this, in situ analysis may be the best option to ensure that the measured isotopic values are accurate.

\section{CONCLUSIONS}

The field of experimental non-traditional stable isotope geochemistry has expanded rapidly in the past decade and is likely to continue to grow as more and more natural fractionations in these isotopic systems are uncovered. Indeed, discoveries of fractionations in geologic and planetary materials thus far have greatly outpaced experimental efforts to quantify fractionation factors capable of explaining such fractionations. The current state of the non-traditional stable isotope field can be understood by considering the difficulties of using trace element geochemistry in a time when almost no partition coefficients had been determined. Therefore, there is a huge opportunity for creative and careful experimentalists to push this new field forward at a rapid pace. But in order to understand what these experiments can tell us we need to be cautious with interpreting the results. Not all experiments can be compared and often times should not be compared. Changing one variable in the experiment is enough to cause a difference in the equilibrium isotope fractionation and users of the data should be aware of this before extrapolating such data beyond its useful range. Therefore, the most useful path forward is to adopt the approach taken by the experimental trace element partitioning community, wherein the 
effects of pressure, temperature, solvent and solute composition, oxygen fugacity, and other potential variables are independently evaluated, and parameterized expressions for isotopic fractionation factors are derived. In this way, the community will move toward the goal of understanding the factors that influence equilibrium isotopic fractionations at the relevant conditions in all systems of geologic interest.

\section{ACKNOWLEDGEMENTS}

A.S. acknowledges NSF Grants EAR1321858 and EAR1464008. We thank the editors, James Watkins, Nicolas Dauphas and Fang-zhen Teng, for the opportunity to contribute to this volume. The chapter was greatly improved by thoughtful reviews from Laura Wasylenki and Mathieu Roskosz.

\section{REFERENCES}

Barling J, Anbar AD (2004) Molybdenum isotope fractionation during adsorption by manganese oxides. Earth Planet Sc Lett 217:315-29,

Beard BL, Handler RM, Scherer MM, Wu LL, Czaja AD, Heimann A, Johnson CM (2010) Iron isotope fractionation between aqueous ferrous iron and goethite. Earth Planet Sc Lett 295:241-250,

Bennett NR, Brenan JM, Fei YW (2015) Metal-silicate Partitioning at High Pressure and Temperature: Experimental Methods and a Protocol to Suppress Highly Siderophile Element Inclusions. Journal of Visualized Experiments:e52725,

Bigeleisen J, Mayer MG (1947) Calculation of Equilibrium Constants for Isotopic Exchange Reactions. J Chem Phys 15:261-267,

Bonnand P, Williams H, Parkinson I, Wood B, Halliday A (2016) Stable chromium isotopic composition of meteorites and metal-silicate experiments: Implications for fractionation during core formation. Earth Planet Sc Lett 435:14-21,

Chiba H, Chacko T, Clayton RN, Goldsmith JR (1989) Oxygen Isotope Fractionations Involving Diopside, Forsterite, Magnetite, and Calcite: Application to Geothermometry. Geochim Cosmochim Ac 53:2985-2995,

Clayton RN, Goldsmith JR, Mayeda TK (1989) Oxygen isotope fractionation in quartz, albite, anorthite and calcite. Geochim Cosmochim Ac 53:725-733,

Clayton RN, Goldsmith JR, Karel KJ, Mayeda TK, Newton RC (1975) Limits on Effect of Pressure on Isotopic Fractionation. Geochim Cosmochim Ac 39:1197-1201,

Corrigan GM (1982) Cooling Rate Studies of Rocks from two Basic Dykes. Mineral Mag 46:387-394,

Dalou C, Koga KT, Shimizu N, Boulon J, Devidal JL (2012) Experimental determination of F and $\mathrm{Cl}$ partitioning between lherzolite and basaltic melt. Contrib Mineral Petr 163:591609 ,

Dauphas N, Roskosz M, Alp E, Golden D, Sio C, Tissot F, Hu M, Zhao J, Gao L, Morris R (2012) A general moment NRIXS approach to the determination of equilibrium Fe 
isotopic fractionation factors: application to goethite and jarosite. Geochim Cosmochim Ac 94:254-275,

Dauphas N, Roskosz M, Alp EE, et al. (2014) Magma redox and structural controls on iron isotope variations in Earth's mantle and crust. Earth Planet Sc Lett 398:127-140,

Deines P, Eggler DH (2009) Experimental determination of carbon isotope fractionation between $\mathrm{CaCO}_{3}$ and graphite. Geochim Cosmochim Ac 73:7256-7274,

Donaldson CH (1976) Experimental Investigation of Olivine Morphology. Contrib Mineral Petr $57: 187-213$,

Elardo SM, Shahar A (2016) Non-chondritic iron isotope ratios in planetary mantles. In Review

Frierdich AJ, Beard BL, Scherer MM, Johnson CM (2014) Determination of the Fe (II) aqmagnetite equilibrium iron isotope fractionation factor using the three-isotope method and a multi-direction approach to equilibrium. Earth Planet Sc Lett 391:77-86,

Hill PS, Schauble EA, Shahar A, Tonui E, Young ED (2009) Experimental studies of equilibrium iron isotope fractionation in ferric aquo-chloro complexes. Geochim Cosmochim Ac 73:2366-2381,

Hin RC, Schmidt MW, Bourdon B (2012) Experimental evidence for the absence of iron isotope fractionation between metal and silicate liquids at $1 \mathrm{GPa}$ and $1250-1300{ }^{\circ} \mathrm{C}$ and its cosmochemical consequences. Geochim Cosmochim Ac 93:164-181,

Hin RC, Fitoussi C, Schmidt MW, Bourdon B (2014) Experimental determination of the Si isotope fractionation factor between liquid metal and liquid silicate. Earth Planet Sc Lett 387:55-66,

Hin RC, Burkhardt C, Schmidt MW, Bourdon B, Kleine T (2013) Experimental evidence for Mo isotope fractionation between metal and silicate liquids. Earth Planet Sc Lett 379:38-48,

Hoering TC (1961) The physical chemistry of isotopic substances: The effect of physical changes on isotope fractionation. Carnegie Inst Wash 60:201-204,

Hu GX, Clayton RN (2003) Oxygen isotope salt effects at high pressure and high temperature and the calibration of oxygen isotope geothermometers. Geochim Cosmochim Ac 67:3227-3246,

Hu MY, Sturhahn W, Toellner TS, Mannheim PD, Brown DE, Zhao JY, Alp EE (2003) Measuring velocity of sound with nuclear resonant inelastic X-ray scattering. Phys Rev B 67 ,

Huang F, Lundstrom C, Glessner J, Ianno A, Boudreau A, Li J, Ferré E, Marshak S, DeFrates J (2009) Chemical and isotopic fractionation of wet andesite in a temperature gradient: experiments and models suggesting a new mechanism of magma differentiation. Geochim Cosmochim Ac 73:729-749,

Icopini GA, Anbar AD, Ruebush SS, Tien M, Brantley SL (2004) Iron isotope fractionation during microbial reduction of iron: The importance of adsorption. Geology 32:205-208,

Johnson CM, Beard BL, Roden EE, Newman DK, Nealson KH (2004) Isotopic constraints on biogeochemical cycling of Fe. Reviews in mineralogy and geochemistry 55:359-408,

Johnson CM, Skulan JL, Beard BL, Sun H, Nealson KH, Braterman PS (2002) Isotopic fractionation between $\mathrm{Fe}(\mathrm{III})$ and $\mathrm{Fe}(\mathrm{II})$ in aqueous solutions. Earth Planet $\mathrm{Sc}$ Lett 195:141-153,

Joy HW, Libby WF (1960) Size Effects among Isotopic Molecules. J Chem Phys 33:1276-1276,

Kempl J, Vroon PZ, Zinngrebe E, van Westrenen W (2013) Si isotope fractionation between Sipoor metal and silicate melt at pressure-temperature conditions relevant to metal segregation in small planetary bodies. Earth Planet Sc Lett 368:61-68, 
Labidi J, Shahar A, Le Losq C, Hillgren V, Mysen B, Farquhar J (2016) Experimentally determined sulfur isotope fractionation between metal and silicate and implications for planetary differentiation. Geochim Cosmochim Ac 175:181-194,

Larsen JF (2005) Experimental study of plagioclase rim growth around anorthite seed crystals in rhyodacitic melt. Am Mineral 90:417-427,

Lazar C, Young ED, Manning CE (2012) Experimental determination of equilibrium nickel isotope fractionation between metal and silicate from $500{ }^{\circ} \mathrm{C}$ to $950{ }^{\circ} \mathrm{C}$. Geochim Cosmochim Ac 86:276-295,

Li W, Beard BL, Li C, Xu H, Johnson CM (2015) Experimental calibration of Mg isotope fractionation between dolomite and aqueous solution and its geological implications. Geochim Cosmochim Ac 157:164-181,

Macris CA, Young ED, Manning CE (2013) Experimental determination of equilibrium magnesium isotope fractionation between spinel, forsterite, and magnesite from 600 to $800^{\circ} \mathrm{C}$. Geochim Cosmochim Ac 118:18-32,

Matsuhisa Y, Goldsmith JR, Clayton RN (1978) Mechanisms of Hydrothermal Crystallization of Quartz at $250{ }^{\circ} \mathrm{C}$ and $15 \mathrm{Kbar}$. Geochim Cosmochim Ac 42:173-184,

Matthews A, Goldsmith JR, Clayton RN (1983) Oxygen Isotope Fractionation between Zoisite and Water. Geochim Cosmochim Ac 47:645-654,

Merrill RB, Wyllie PJ (1973) Absorption of iron by platinum capsules in high pressure rock melting experiments. Am Mineral 58:16-20,

Mills RD, Glazner AF (2013) Experimental study on the effects of temperature cycling on coarsening of plagioclase and olivine in an alkali basalt. Contrib Mineral Petr 166:97111 ,

Murphy CA, Jackson JM, Sturhahn W (2013) Experimental constraints on the thermodynamics and sound velocities of hcp-Fe to core pressures. Journal of Geophysical Research: Solid Earth 118:1999-2016,

Nakada R, Takahashi Y, Tanimizu M (2013) Isotopic and speciation study on cerium during its solid-water distribution with implication for Ce stable isotope as a paleo-redox proxy. Geochim Cosmochim Ac 103:49-62,

Northrop DA, Clayton RN (1966) Oxygen-Isotope Fractionations in Systems Containing Dolomite. J Geol 74:174-\&,

O'Neil JR (1986) Theoretical and Experimental Aspects of Isotopic Fractionation. Rev Mineral $16: 1-40$,

Pattison DRM (1994) Are Reversed Fe-Mg Exchange and Solid-Solution Experiments Really Reversed? Am Mineral 79:938-950,

Poitrasson F, Roskosz M, Corgne A (2009) No iron isotope fractionation between molten alloys and silicate melt to $2000{ }^{\circ} \mathrm{C}$ and $7.7 \mathrm{GPa}$ : Experimental evidence and implications for planetary differentiation and accretion. Earth Planet Sc Lett 278:376-385,

Polyakov VB (1998) On anharmonic and pressure corrections to the equilibrium isotopic constants for minerals. Geochim Cosmochim Ac 62:3077-3085,

Polyakov VB (2009) Equilibrium Iron Isotope Fractionation at Core-Mantle Boundary Conditions. Science 323:912-914,

Polyakov VB, Kharlashina NN (1994) Effect of Pressure on Equilibrium Isotopic Fractionation. Geochim Cosmochim Ac 58:4739-4750, 
Polyakov VB, Clayton RN, Horita J, Mineev SD (2007) Equilibrium iron isotope fractionation factors of minerals: Reevaluation from the data of nuclear inelastic resonant X-ray scattering and Mossbauer spectroscopy. Geochim Cosmochim Ac 71:3833-3846,

Polyakov VB, Mineev SD, Clayton RN, Hu G, Mineev KS (2005) Determination of tin equilibrium isotope fractionation factors from synchrotron radiation experiments. Geochim Cosmochim Ac 69:5531-5536,

Richter FM, Watson EB, Mendybaev RA, Teng FZ, Janney PE (2008) Magnesium isotope fractionation in silicate melts by chemical and thermal diffusion. Geochim Cosmochim Ac 72:206-220,

Roskosz M, Luais B, Watson HC, Toplis MJ, Alexander CMO, Mysen BO (2006) Experimental quantification of the fractionation of $\mathrm{Fe}$ isotopes during metal segregation from a silicate melt. Earth Planet Sc Lett 248:851-867,

Roskosz M, Sio CKI, Dauphas N, Bi WL, Tissot FLH, Hu MY, Zhao JY, Alp EE (2015) Spinelolivine-pyroxene equilibrium iron isotopic fractionation and applications to natural peridotites. Geochim Cosmochim Ac 169:184-199,

Schauble EA (2004) Applying stable isotope fractionation theory to new systems. Rev Mineral Geochem 55:65-111,

Schuessler JA, Schoenberg R, Behrens H, von Blanckenburg F (2007) The experimental calibration of the iron isotope fractionation factor between pyrrhotite and peralkaline rhyolitic melt. Geochim Cosmochim Ac 71:417-433,

Shahar A, Young ED, Manning CE (2008) Equilibrium high-temperature $\mathrm{Fe}$ isotope fractionation between fayalite and magnetite: An experimental calibration. Earth Planet Sc Lett 268:330-338,

Shahar A, Ziegler K, Young ED, Ricolleau A, Schauble EA, Fei YW (2009) Experimentally determined $\mathrm{Si}$ isotope fractionation between silicate and $\mathrm{Fe}$ metal and implications for Earth's core formation. Earth Planet Sc Lett 288:228-234,

Shahar A, Hillgren VJ, Young ED, Fei YW, Macris CA, Deng LW (2011) High-temperature Si isotope fractionation between iron metal and silicate. Geochim Cosmochim Ac 75:76887697 ,

Shahar A, Hillgren VJ, Horan MF, Mesa-Garcia J, Kaufman LA, Mock TD (2015) Sulfurcontrolled iron isotope fractionation experiments of core formation in planetary bodies. Geochim Cosmochim Ac 150:253-264,

Shahar A, Schauble EA, Caracas R, Gleason AE, Reagan MM, Xiao Y, Shu J, Mao W (2016) Pressure-dependent isotopic composition of iron alloys. Science In Press,

Skulan JL, Beard BL, Johnson CM (2002) Kinetic and equilibrium Fe isotope fractionation between aqueous Fe(III) and hematite. Geochim Cosmochim Ac 66:2995-3015,

Sturhahn W (2000) CONUSS and PHOENIX: Evaluation of nuclear resonant scattering data. Hyperfine Interact 125:149-172,

Sturhahn W, Toellner TS, Alp EE, Zhang X, Ando M, Yoda Y, Kikuta S, Seto M, Kimball CW, Dabrowski B (1995) Phonon Density-of-States Measured by Inelastic Nuclear Resonant Scattering. Phys Rev Lett 74:3832-3835,

Urey HC (1947) The Thermodynamic Properties of Isotopic Substances. J Chem Soc:562-581,

Wasylenki LE, Swihart JW, Romaniello SJ (2014) Cadmium isotope fractionation during adsorption to Mn oxyhydroxide at low and high ionic strength. Geochim Cosmochim Ac 140:212-26, 
Wasylenki LE, Howe HD, Spivak-Birndorf LJ, Bish DL (2015) Ni isotope fractionation during sorption to ferrihydrite: Implications for $\mathrm{Ni}$ in banded iron formations. Chem Geol 400:56-64,

Welch SA, Beard BL, Johnson CM, Braterman PS (2003) Kinetic and equilibrium Fe isotope fractionation between aqueous Fe(II) and Fe(III). Geochim Cosmochim Ac 67:42314250 ,

Williams HM, Wood BJ, Wade J, Frost DJ, Tuff J (2012) Isotopic evidence for internal oxidation of the Earth's mantle during accretion. Earth Planet Sc Lett 321:54-63,

Williams HM, McCammon CA, Peslier AH, Halliday AN, Teutsch N, Levasseur S, Burg JP (2004) Iron isotope fractionation and the oxygen fugacity of the mantle. Science 304:1656-1659,

Young ED, Galy A, Nagahara H (2002) Kinetic and equilibrium mass-dependent isotope fractionation laws in nature and their geochemical and cosmochemical significance. Geochim Cosmochim Ac 66:1095-1104,

Young ED, Manning CE, Schauble EA, Shahar A, Macris CA, Lazar C, Jordan M (2015) Hightemperature equilibrium isotope fractionation of non-traditional stable isotopes: Experiments, theory, and applications. Chem Geol 395:176-195,

\section{FIGURE CAPTIONS}

Figure 1. A schematic of a multi-direction approach experiment showing two phases ('A' and ' $\mathrm{B}$ ') that have starting fractionation values $\left(\Delta^{\mathrm{i}} \mathrm{E}_{\mathrm{A}-\mathrm{B}}\right)$ lying on opposite sides of the equilibrium value. As the experimental duration progresses the values bracket the true equilibrium value. Here ' $i$ ' represents an arbitrary isotope of element ' $\mathrm{E}$ ', and $\Delta^{\mathrm{i}} \mathrm{E}_{\mathrm{A}-\mathrm{B}}=\delta^{\mathrm{i}} \mathrm{E}_{\mathrm{A}}-\delta^{\mathrm{i}} \mathrm{E}_{\mathrm{B}}$.

Figure 2. A schematic of the three-isotope exchange method shown for arbitrary isotopes 'i', ' $\mathrm{j}$ ', and ' $x$ ', of element ' $E$ '. Figure 2A depicts the more traditional path during a dissolution and precipitation experiment. Phase 1 (red circles) is unspiked and starts on the terrestrial fractionation. Over time, as the system evolves towards equilibrium, Phase 1 moves to lighter values, while Phase 2 (blue squares), which is spiked so that is starts off of the terrestrial fractionation line, moves towards heavier values. The yellow star represents the bulk value of the system. Equilibrium is reached when both phases reach the secondary fractionation line, though in some cases the phases may not fully reach the equilibrium values, as depicted by the transparent symbols. In such cases, the equilibrium fractionation is determined by extrapolation. Figure $2 \mathrm{~B}$ depicts a higher temperature experiment where all the isotopes first mix at the bulk value for the system and then 'unmix' along the secondary fractionation line. In this scenario a time series is also needed to prove equilibrium.

Figure 3. Schematics of A) a controlled-atmosphere metal loop experiment, B) a piston cylinder experiment (after Young et al. 2015), C) a multi-anvil experiment (after Bennett et al. 2015) and D) an NRIXS experiment (after Dauphas et al. 2012). A) A cross-sectional view of the internal arrangement of a typical ambient pressure controlled-atmosphere wire loop experiment. Typical gas mixtures consist of $\mathrm{CO}-\mathrm{CO}_{2}$ and $\mathrm{H}_{2}-\mathrm{CO}_{2}$, though other mixtures are sometimes used. Wire loops are typically $\mathrm{Pt}$ or Re. B) A cross-sectional view of a typical piston cylinder experiment 
showing where the capsule resides and typical assembly materials. C) An external view of a typical multi-anvil ceramic octahedron and a cross-sectional view of the internal arrangement of the octahedron. D) A typical NRIXS experimental set-up. Incoming synchrotron x-ray pulses (separated by $153 \mathrm{nsec}$ ) first pass through the diamond pre-monochromator (PM), and then through the Si channel-cut high resolution monochromator (HRM). The beam is focused by small horizontal $(20-\mathrm{cm})$ and vertical $(10-\mathrm{cm})$ Kirkpatrick-Baez $(\mathrm{KB})$ mirrors onto the DAC sample. The forward synchrotron Mössbauer spectroscopy (SMS) signal is collected through the diamond axis on an avalanche photodiode (APD). Three additional side APDs collect the NRIXS signal through the $\mathrm{X}$-ray transparent gasket. 


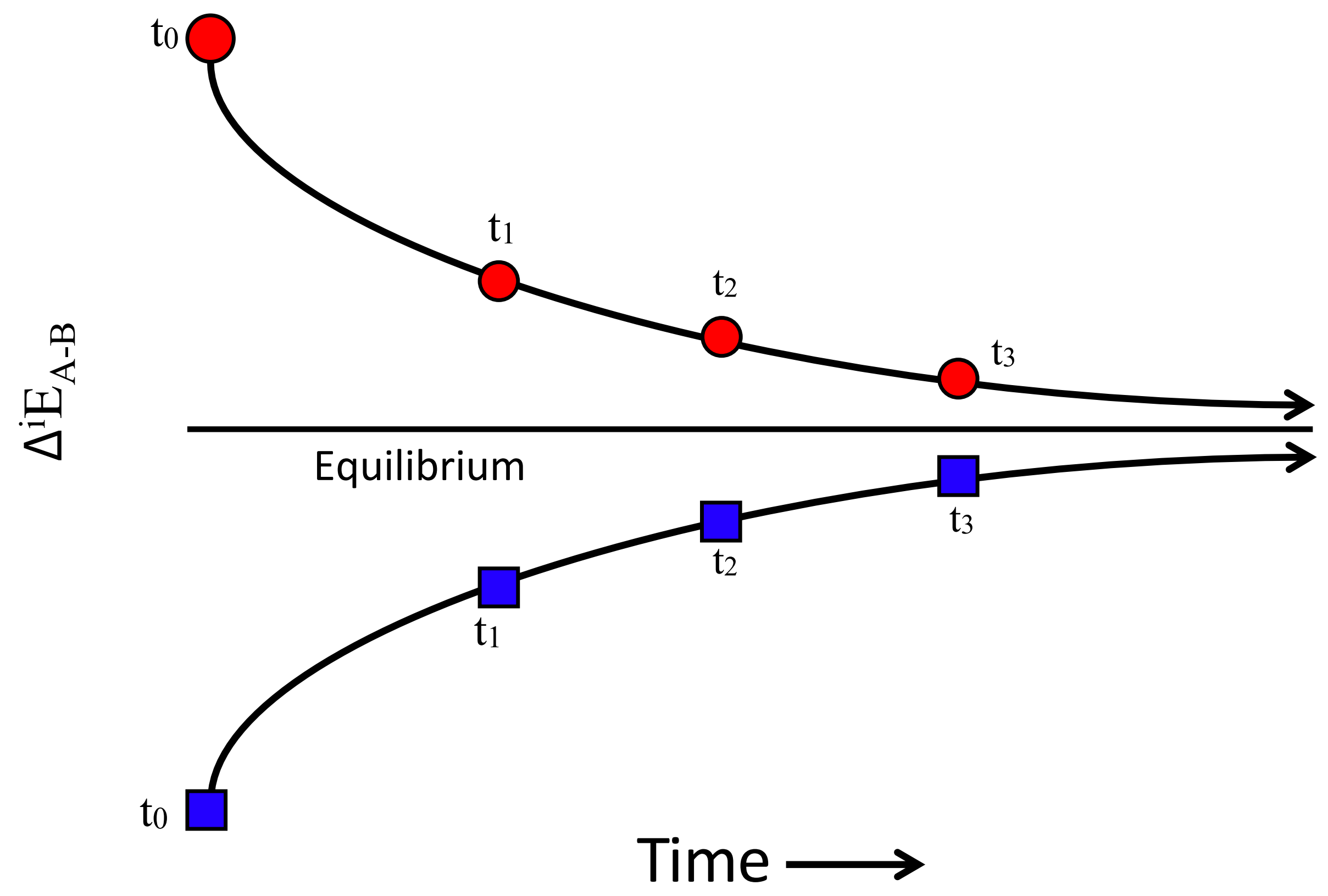



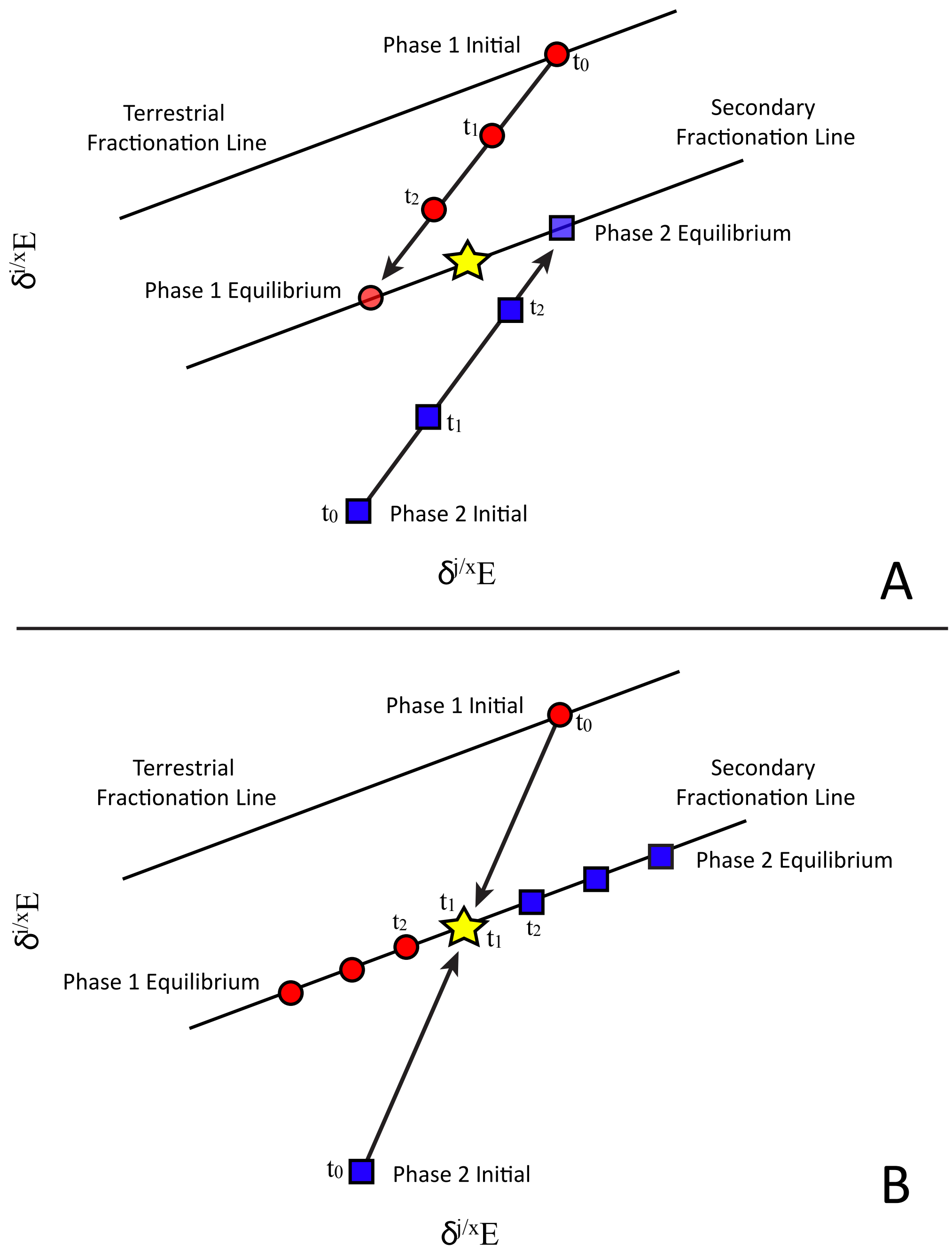

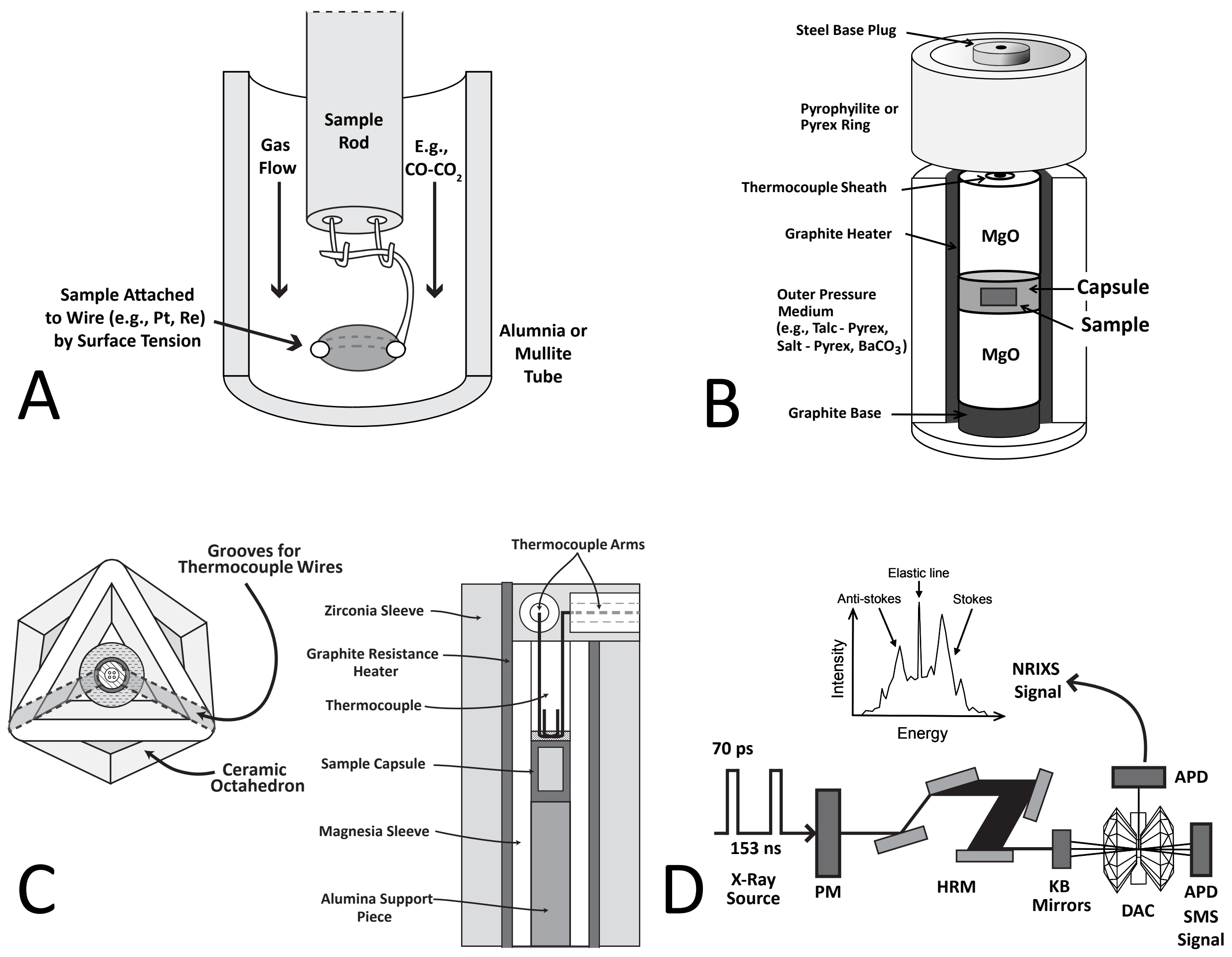